\title{
Electrodos metálicos recubiertos de PEDOT para detectar Arsénico. Caso de medios acuosos
}

\section{PEDOT coated metallic electrodes to detect Arsenic. Case of aqueous media}

\author{
Hayden Phillips Brenes ${ }^{1}$ \\ Diana Robles Chaves ${ }^{2}$ \\ Ricardo Starbird Pérez ${ }^{3}$ \\ Tecnológico de Costa Rica, Costa Rica
}

\begin{abstract}
Resumen. La contaminación del agua con arsénico (As) se ha convertido en una de las principales preocupaciones para la salud pública. La disminución gradual del suministro de agua ha obligado a las autoridades a adoptar programas de protección para proteger las fuentes de agua limpia para el consumo humano y de uso productivo. La toma de decisiones acerca de las fuentes de agua depende del análisis más rápido y preciso de los datos bajo estudio. Las herramientas innovadoras, como los sensores de tipo medición in situ son opciones prometedoras en esta disciplina, debido a su bajo costo y mínimo impacto ambiental. Este documento describe el diseño, construcción y caracterización de un electrodo de oro recubierto con polímero PEDOT, para medir As en medio acuoso. El funcionamiento del electrodo se verificó midiendo concentraciones de As utilizando la técnica de voltametría cíclica, que permite observar picos específicos de corriente eléctrica característicos para cada nivel de concentración del contaminante. Los resultados experimentales indicaron una sensibilidad máxima del electrodo para una concentración de $66 \mu \mathrm{g} / \mathrm{L}$ y un rango de medición lineal a concentraciones desde $166 \mu \mathrm{g} / \mathrm{L}$ a $1500 \mu \mathrm{g} / \mathrm{L}$. Además, la medición se simuló en el software de simulación multifísico COMSOL, para comparar los resultados experimentales con los resultados teóricos computacionales.
\end{abstract}

Palabras clave. Arsénico, voltametría cíclica, PEDOT, electrodos, microfabricación

\begin{abstract}
Water pollution with arsenic (As) has become a major public health issue. The gradual reduction of the water supply has forced authorities to adopt protection programs for clean water sources for human consumption and productive use. The decision making about water sources depends on the faster and more accurate analysis of the data under study. Innovative tools such as in situ measurement type sensors are promising options in this discipline because of their low cost and minimal environmental impact. This document describes the design, construction and characterization of a gold microelectrode coated with PEDOT polymer, to measure As in aqueous medium. The operation of the electrode was verified by measuring As concentrations using the technique of cyclic voltammetry, which allows to observe specific peaks of electrical current characteristic for each level of concentration of the contaminant. The experimental results indicated a maximum sensitivity of the electrode for a concentration of 66ug / L and a linear measurement range of $166 \mathrm{ug}$ / L to 1500ug / L. Furthermore, the measurement was simulated in the COMSOL multiphase simulation software to compare the experimental results with the theoretical computational results.
\end{abstract}

Keywords. Arsenic, cyclic-voltammetry, PEDOT, electrodes, micro-fabrication

\footnotetext{
${ }^{1}$ ÁProfesor e investigador de la Escuela de Ingeniería Electrónica y del Centro de Investigación y de Servicios Químicos y Microbiológicos (CEQIATEC) del Tecnológico de Costa Rica, Cartago, Costa Rica. Dirección electrónica: hphillips@itcr.ac.cr

${ }^{2}$ Química regente del Centro de Investigación y de Servicios Químicos y Microbiológicos (CEQIATEC), Tecnológico de Costa Rica. Dirección electrónica: drobles@itcr.ac.cr

${ }^{3}$ Químico Industrial, profesor e investigador de la Escuela de Química y del Centro de Investigación y de Servicios Químicos y Microbiológicos (CEQIATEC), Tecnológico de Costa Rica, Cartago, Costa Rica. Dirección electrónica: $\underline{\text { rstarbird@itcr.ac.cr }}$
} 


\section{Introducción}

En Costa Rica, el agua para uso humano y animal se ha visto afectada debido a la presencia de arsénico sobre los límites máximos permisibles para el consumo humano en varias fuentes y afluentes, principalmente en Cañas, Bagaces, San Carlos y algunos otros pueblos agrícolas de Guanacaste y Alajuela, en el noroeste del país, según la Organización Mundial de la Salud (Defensoría de los Habitantes de la República, 2014; Mora-Alvarado, Urbina-Campos, y
Chamizo-García, 2015). Las áreas afectadas (ver Figura 1) coinciden con muchas de las granjas extensivas de piña que todavía usan compuestos herbicidas antropogénicos de arsénico, como el ácido monometilarsónico (MMA) y el ácido dimetilarsínico (DMA) (Tanaka et al., 2013). Es importante señalar que la producción extensiva de piña de Costa Rica se concentra en Buenos Aires, San Carlos, Siquirres, Sarapiquí y Esparza, no así en la provincia de Guanacaste (Quijandria, Berrocal y Pratt, 1997).

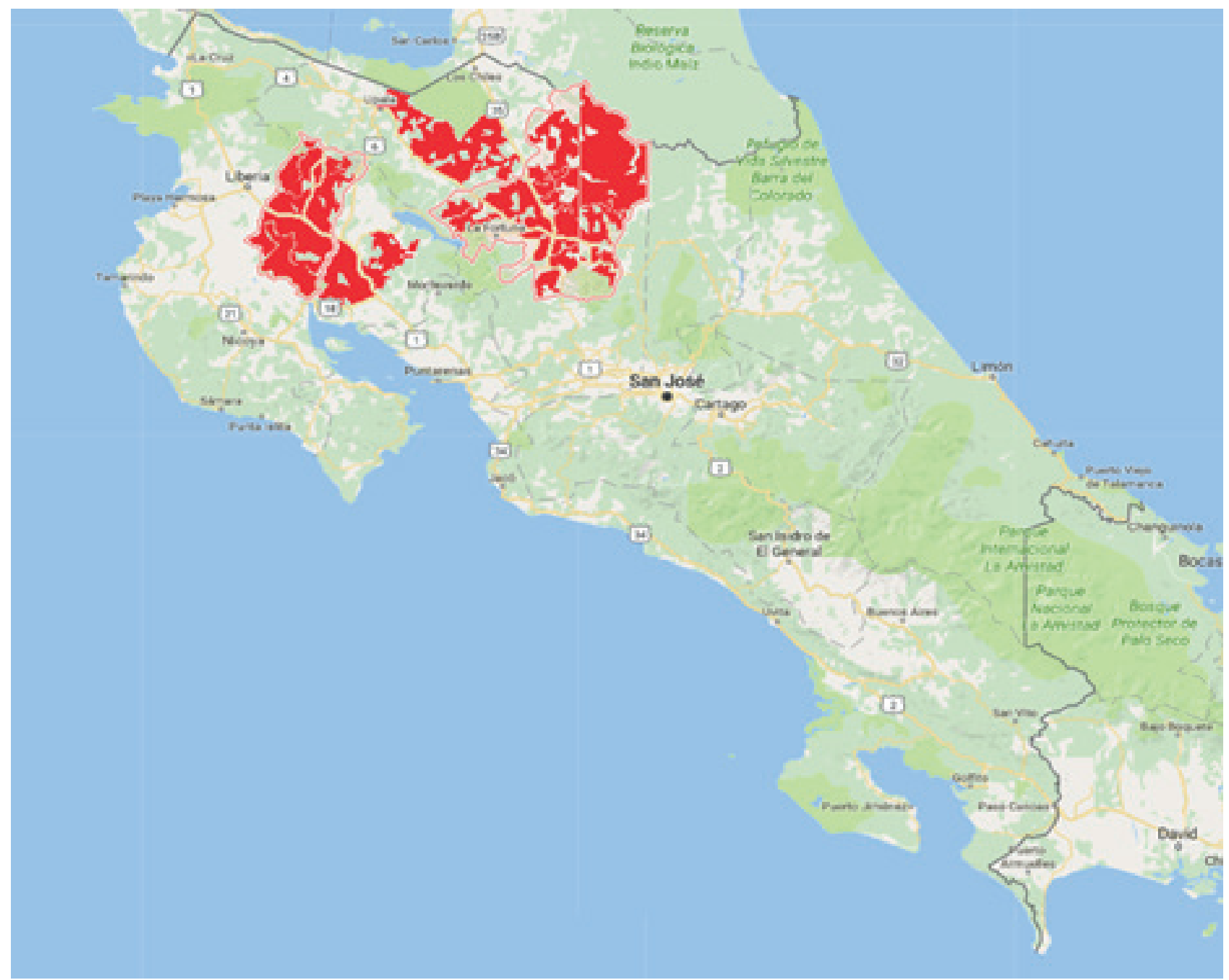

Figura 1. Áreas de Costa Rica afectadas (en rojo) debido a la presencia de componentes de arsénico. Fuente: Defensoria de los Habitantes de la República, 2014 
El arsénico se puede encontrar en pequeños depósitos en forma natural en la corteza terrestre y es susceptible a distribuirse en el medio ambiente por el aire, el agua y la tierra. Es altamente tóxico cuando se encuentra en su forma inorgánica ( Defensoria de los Habitantes de la República, 2014). Además, puede existir en cuatro estados de valencia: -3, 0, +3 y +5 (Quansah et al., 2015; World Health Organization, 2004; Gomez-Caminero et al., 2000).

El arsénico inorgánico soluble es altamente tóxico. El consumo de arsénico inorgánico durante un período prolongado puede provocar intoxicación crónica (arsenicosis). Los compuestos orgánicos de arsénico, abundantes en algunos alimentos como los mariscos, son menos nocivos para la salud y son rápidamente eliminados por el cuerpo (World Health Organization, 2004; 2010; Gomez-Caminero et al., 2000).

La detección y cuantificación de arsénico se ha realizado de manera tradicional, utilizando métodos tales como espectrometría ultravioletavisible (UV-Vis), espectrometría de absorción atómica con generación de hidruro (HGAAS), absorción atómica con horno de grafito, espectroscopia de emisión con plasma inductivo de argón, espectrometría de masas con plasma inductivamenteacoplado,especiacióndearsénico mediante técnicas acopladas o espectrometría de fluorescencia de rayos $X$ de energía dispersiva (Litter, Armienta y Farias, 2009; Morand, Giménez, Benitez y Garro, 2008). Estos métodos no permiten realizar mediciones in situ debido a que requieren de equipos especializados de alto costo y tamaño, un volumen alto de muestras, condiciones controladas de laboratorio, curvas de calibración previas y reactivos específicos para su preparación.

La detección de arsénico en medio acuoso mediante análisis electroquímico in situ, utilizando electrodos como transductores de señales químicas, requiere de menor tiempo y recursos que otros métodos de detección tradicionales. De esta manera, la respuesta de las autoridades de salud puede ser más expedita, especialmente en áreas remotas donde los laboratorios químicos no pueden establecerse a corto plazo.

Se han realizado estudios previos con el uso de electrodos para la detección electroquímica de arsénico tales como electrodos de carbono modificado con vidrio con nanopartículas de platino para la detección de As (III), utilizando la voltametría cíclica y la voltametría de barrido lineal. Este experimento fue limitado por la interferencia de $\mathrm{C}_{\mathrm{u} 3} \mathrm{~A}_{\mathrm{s} 2}$ debido a la separación de cobre (Dai y Compton, 2006). Asimismo, se utilizó un electrodo de carbono vítreo modificado con nanopartículas de óxido de cobalto para medir As (III) mediante voltametría cíclica, obteniendo una sensibilidad máxima de $11 \mathrm{nmol}$ / L (Salimi, Mamkhezri, Hallaj, \& Soltanian, 2008). También se han llevado a cabo estudios con electrodos modificados con películas de polímeros conductores, tales como un electrodo de carbono vítreo recubierto con película PEDOT para controlar las concentraciones de arsénico superiores a $500 \mu \mathrm{mol} / 1$ en presencia de yoduro (Chen, Tsai, Chen, \& Lin, 2011).

Los electrodos utilizados en los estudios mencionados previamente se fabricaron sobre sustratos rígidos, lo que restringe el tipo de celda electroquímica en cuanto a su tamaño y forma, al igual que el tipo de muestra para la medición.

Este documento describe la fabricación de un electrodo de oro depositado sobre un sustrato de poliimida, pasivado con fotoprotector y recubierto con polímero conductivo de poli (3,4-etilendioxitiofeno) (PEDOT). El sustrato utilizado le brinda alta flexibilidad y portabilidad, con un alto rendimiento para detección de arsénico en un medio acuoso por medio de voltametría cíclica en un ambiente 
controlado de laboratorio, con posibles aplicaciones para detección y cuantificación de contaminantes in situ. Los resultados de la medición experimental se compararon con los obtenidos en una simulación multifísica, que se alimentó de parámetros obtenidos de manera experimental.

\section{Metodología}

\section{Materiales}

Monómero 3,4-etilendioxitiofeno (EDOT) y tensioactivo poliestireno sulfonato de sodio (NaPSS) (PM=70000 g/mol) adquiridos en SigmaAldrich, se usaron sin purificación adicional para crear la suspensión de monómero EDOT que se depositaría sobre el oro para recubrirlo. Cloruro de potasio $(\mathrm{KCl})$, calidad analítica, de Merck se utilizó en el electrodo de referencia para la voltamería cíclica. El fotorresistivo positivo S-1805 que se utilizó en la pasivación del electrodo y el sustrato de poliimida que funcionó como base del electrodo se adquirieron de Hauque Enterprises y American Durafilm, respectivamente.

\section{Deposición de oro sobre el sustrato de poliimida}

El oro que conforma las pistas conductoras del electrodo se depositó sobre el sustrato de poliimida por medio de la técnica de rociado (sputtering). El espesor de la capa depositada fue de alrededor de $60 \mathrm{~nm}$. La resistencia eléctrica de la deposición para corriente continua (CC) fue de 15 ohmios aproximadamente (Hayt, Kemmerly, \& Durbin, 1986).

\section{Pasivación con fotorresistivo}

El área completa del electrodo se pasivó con fotorresistivo S1805, recubriéndolo por centrifugación para lograr un espesor plano de $500 \mathrm{~nm}$. Posteriormente, se removió químicamente un área circular de $0.0113 \mathrm{~cm}^{2}$ donde el oro quedó expuesto para ser utilizado como el área activa del electrodo. Finalmente, el electrodo se curó a $180^{\circ} \mathrm{C}$ para mejorar la respuesta de pasivación ante ataques químicos.

\section{Polimerización del PEDOT sobre el oro}

Para mejorar la capacidad de carga ante el fenómeno de oxidación-reducción en el proceso de voltametría cíclica, el área activa del electrodo se polimerizó por electrodeposición. El polímero poli (3,4-etilendioxitiofeno) (PEDOT) se depositó a partir de su forma monomérica, suspendida en un medio micelar de poliestirenosulfonato sódico (NaPSS). NaPSS funciona como surfactante, lo que permite manejar el EDOT como una suspensión dentro una fase acuosa. La suspensión PEDOT/NaPSS se electropolimerizó a partir de una concentración de monómero (EDOT) en NapsS de $2.0 \mathrm{~g} / 100 \mathrm{ml}$ en condiciones galvanostáticas con una densidad de carga fija de $240 \mathrm{mC} / \mathrm{cm}^{2}$ de acuerdo con trabajos previos que demuestran que esta densidad de carga se encuentra dentro de un rango óptimo para efectos de capacidad de carga (Starbird, Bauhofer, Meza-Cuevas, \& Krautschneider, 2012).

Medición de concentración de arsénico por voltametría cíclica (CV)

El método de voltametría cíclica permite cuantificar el arsénico en disolución midiendo la transferencia de electrones en una reacción electroquímica. Para realizar el estudio, se aplica una tensión eléctrica entre dos electrodos, que cambia su magnitud en un rango definido, aumentando y disminuyendo de manera cíclica.

Simultáneamente, se mide la corriente eléctrica entre los electrodos; cuando se produce un proceso de oxidación o reducción de la especie bajo estudio, se produce un pico de corriente que se detecta y se cuantifica. La magnitud del pico de corriente es proporcional a la concentración de la especie en la disolución. La Figura 2 muestra las curvas de corriente debidas a la oxidación para varias concentraciones de arsénico (Kumar Jena, \& Retna Raj, 2008). Las señales de corriente de 


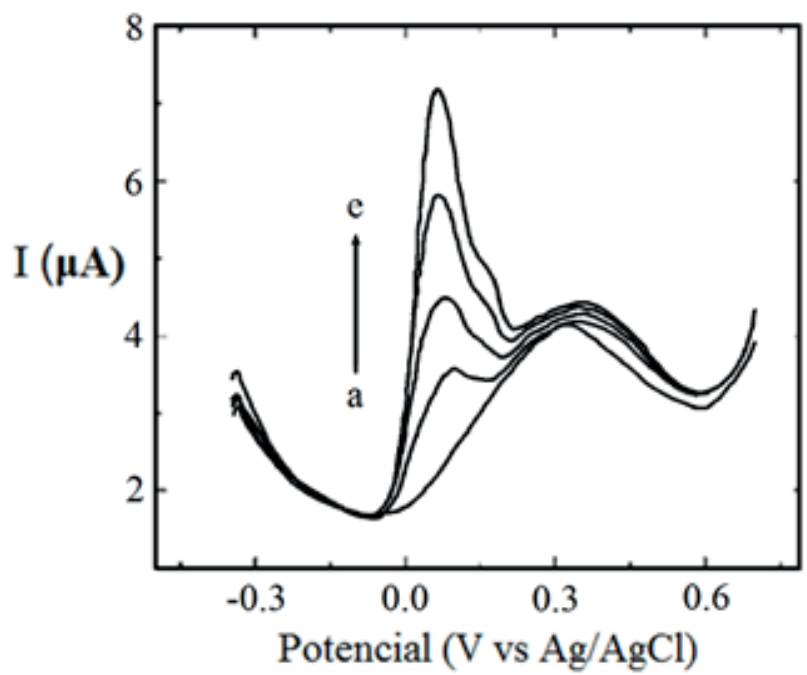

Figura 2. Respuesta de oxidación del arsénico en un análisis de voltametría cíclica para diferentes concentraciones en medio acuoso. Fuente: Kumar Jena y Retna Raj, 2008

la figura para cada concentración se encuentran entre el rango de tensión de 0 a 0,1 V.

Los picos de corriente que se obtienen del estudio de voltametría cíclica se deben al intercambio de electrones entre el electrodo y la especie de arsénico, al producirse un desplazamiento de las cargas desde la especie hacia el electrodo (oxidación) o desde el electrodo hacia la especie (reducción), dependiendo si la diferencia de potencial eléctrico electrodoespecie es positiva o negativa respectivamente (Andrienko, 2008).

El proceso de reducción-oxidación se rige por la Ley de Difusión de Fick, definida en las ecuaciones (1) y (2) (Andrienko, 2008).

$$
\begin{aligned}
& \frac{\delta C_{O x}(x, t)}{\delta t}=D_{O x} \frac{\delta^{2} C_{O x}(x, t)}{\delta^{2} x} \\
& \frac{\delta C_{R e d}(x, t)}{\delta t}=D_{\text {Red }} \frac{\delta^{2} C_{R e d}(x, t)}{\delta^{2} x}
\end{aligned}
$$

Donde $\mathrm{D}_{\mathrm{Ox}} \mathrm{y} \mathrm{D}_{\text {Red }}$ corresponden a los coeficientes de oxidación y reducción respectivamente.

\section{Preparación de muestras}

Las disoluciones se prepararon a partir de un estándar certificado de trióxido de arsénico (material puro) en ácido nítrico $\left(\mathrm{HNO}_{3}\right)$ al $2 \%$, distribuido por la compañía Sigma Aldrich. Cada muestra se combinó con una disolución de cloruro de potasio $(\mathrm{KCl}) 1$ molar en una proporción de $16 \mathrm{~mL}$ de disolución de arsénico por $8 \mathrm{~mL}$ de $\mathrm{KCl} 1$ molar. Esta combinación de disoluciones mejora la fuerza iónica para efectos de la medición de voltametría cíclica.

Para determinar la máxima sensibilidad del electrodo se midió dinámicamente, mediante voltametría cíclica, una disolución de arsénico con una concentración de $25 \mu \mathrm{g} / \mathrm{L}$ que se incrementó gradualmente hasta que se obtuvo una lectura mayor al ruido del aparato de medición. Esta lectura se logró a partir de una concentración de arsénico de $66 \mu \mathrm{g} / \mathrm{L}$. Posteriormente, se determinó la zona lineal del 
comportamiento del electrodo, que se encontró a partir de $166 \mu \mathrm{g} / \mathrm{L}$. Las concentraciones de las disoluciones de arsénico para analizar la zona de comportamiento lineal del electrodo se muestran en la Tabla 1. El pH para toda la disolución fue de aproximadamente 2.5 .

Para realizar el análisis de voltametría cíclica se utilizó un Potenciostato/Galvanostato modular de alto rendimiento de la marca Metrohm Autolab, modelo PGSTAT128N, con una velocidad fija de barrido de potencial eléctrico de $0.05 \mathrm{~V} / \mathrm{s}$, un potencial inicial (límite inferior) de $-0.5 \mathrm{~V}$ y un potencial final (límite superior) de $0.8 \mathrm{~V}$. Como electrodo de referencia, se usó el tipo de cloruro de plata y plata $(\mathrm{Ag} / \mathrm{AgCl})$ con una solución salina de cloruro de potasio $(\mathrm{KCl})$ 3 molar, que tiene un potencial formal de celda media de $0.235 \mathrm{~V}$ (Andrienko, 2008). Como electrodo de conteo se utilizó una lámina de platino de dimensiones de $1.5 \times 1.5 \mathrm{~cm}$.

Tabla 1

Concentraciones de Arsénico

\begin{tabular}{lc}
\hline Muestra & $\begin{array}{c}\text { Disolución de Arsénico } \\
\mu \mathrm{g} / \mathrm{L}( \pm 1.4)\end{array}$ \\
\hline 1 & 166 \\
2 & 333 \\
3 & 506 \\
4 & 666 \\
5 & 840 \\
6 & 1000 \\
\hline
\end{tabular}

\section{Resultados}

\section{Experimento de Voltametría Ciclica}

A partir de la medición ciclo voltamétrica se obtuvo un pico de corriente para cada muestra de arsénico analizada, como se muestra en la Figura 3. Los picos de corriente eléctrica correspondientes al proceso de oxidación para cada concentración medida se ubicaron cerca de los $0 \mathrm{~V}$ de tensión eléctrica. Estos resultados son consistentes con los obtenidos en análisis previos de disoluciones de la misma especie (Kumar Jena \& Retna Raj, 2008).

Los datos obtenidos se muestran en la Tabla 2. La figura 4 muestra una relación lineal entre el valor del pico de corriente y la concentración de la especie que se deduce a partir de los resultados obtenidos.

Para confirmar la dependencia de los picos de corriente de oxidación y la concentración de arsénico, se realizó una prueba estadística tipo ANOVA que permite establecer si existe causalidad entre los datos bajo análisis. El número estadístico $\mathrm{F}$ arrojado en la prueba fue de 7.56, el número $\mathrm{F}$ crítico fue de 2.44 y el valor $\mathrm{P}$ fue de $4 \times 10^{-5}(\alpha=0.01)$. Estos números indican que la corriente máxima realmente es proporcional a la concentración de arsénico en la disolución (Rodríguez y Desan, 2016; Vicéns Otero, Herrarte Sánchez y Medina Moral, 2005). 
Electrodos metálicos recubiertos de PEDOT para detectar Arsénico

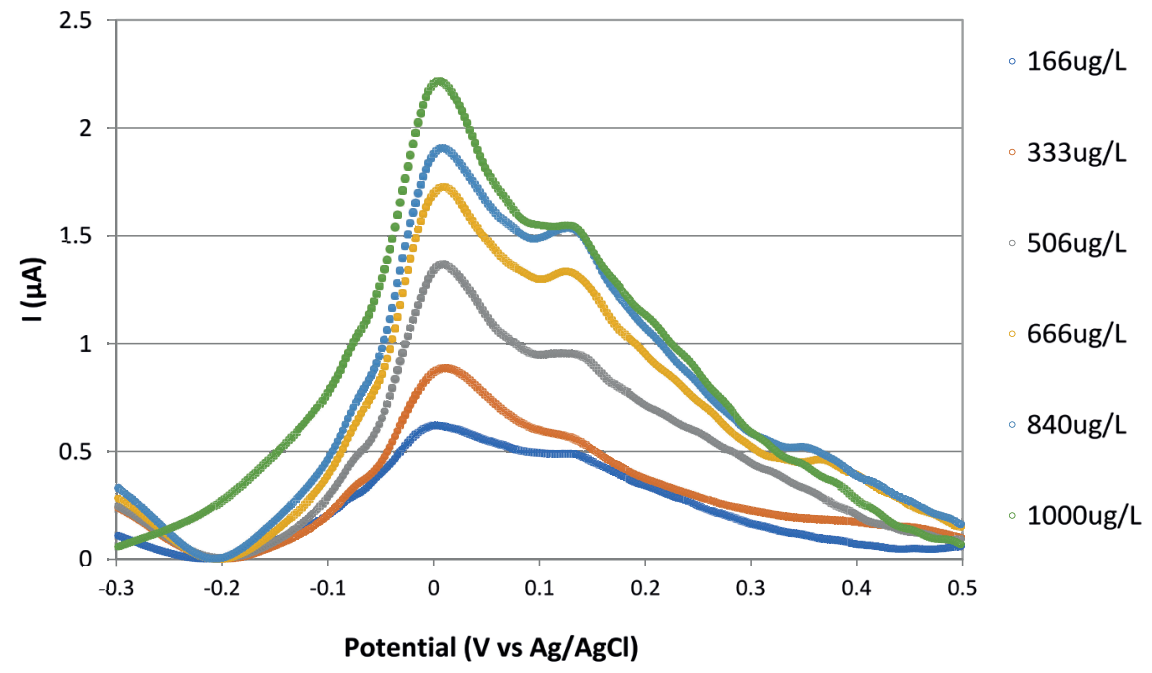

Figura 3. Curvas experimentales de ciclo voltametría para diferentes concentraciones de arsénico: Pico de corriente $(\mu \mathrm{A})$ vs. Potencial eléctrico(V)

Tabla 2

Amplitud de picos de corriente por $\mathrm{CV}$

\begin{tabular}{ccc}
\hline $\begin{array}{c}\text { Concentración de la muestra } \\
\mu \mathrm{g} / \mathrm{L}( \pm 1.4)\end{array}$ & $\begin{array}{c}\text { Pico de corriente } \\
\mu \mathrm{A}( \pm 0.01)\end{array}$ & $\begin{array}{c}\text { Desviación Estándar } \\
\mu \mathrm{A}\end{array}$ \\
\hline 166 & 0.72 & 0.34 \\
333 & 1.00 & 0.53 \\
506 & 1.10 & 0.40 \\
666 & 1.59 & 0.43 \\
840 & 1.82 & 0.38 \\
1000 & 2.01 & 0.35 \\
\hline
\end{tabular}

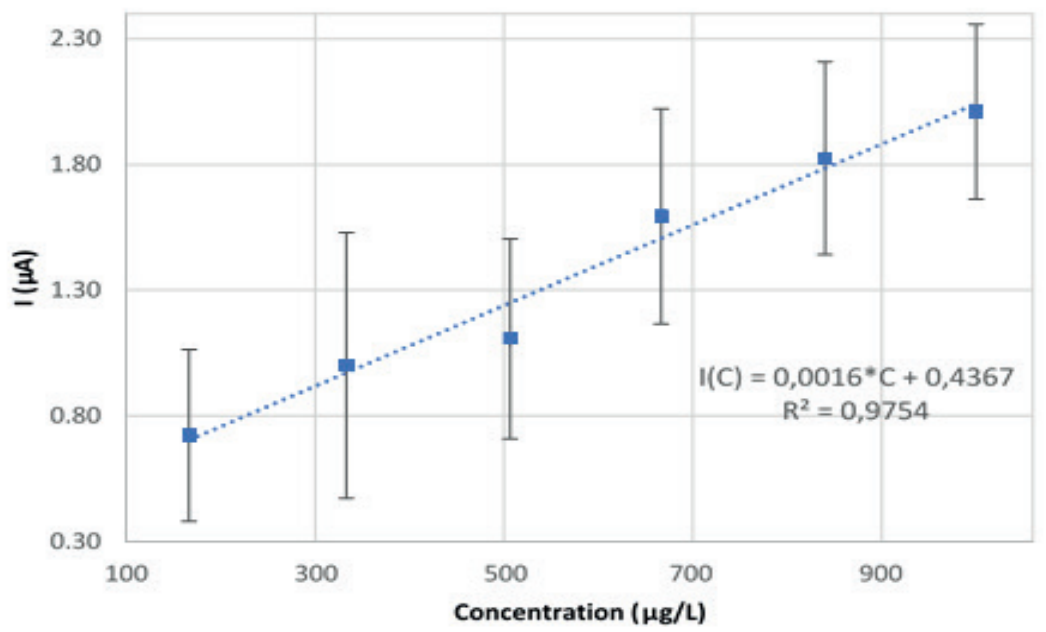

Figura 4. Picos de corriente de oxidación de arsénico obtenidos por voltametría cíclica. Pico de corriente $(\mu \mathrm{A})$ vs Concentración de arsénico $(\mu \mathrm{g} / \mathrm{L})$. Resultados experimentales. 
A partir de los datos en la tabla II, se calculó el coeficiente de difusión para el arsénico bajo las condiciones controladas de laboratorio, con un valor obtenido de $6,7 \times 10^{-5} \mathrm{~m}^{2} / \mathrm{s}$. El cálculo se realizó utilizando la ecuación de Randles-Sevcik (Rieger, 1994).

$$
i_{P}=0,4463 \cdot n \cdot F \cdot A \cdot C\left(\frac{n \cdot F \cdot v \cdot D}{R \cdot T}\right)^{1 / 2}
$$

Donde iP es el pico de corriente medido, $n$ es el número de electrones transferidos en el proceso de oxidación-reducción, A es el área del electrodo de prueba, $\mathrm{F}$ es la constante de Faraday, D es el coeficiente de difusión de la especie, $C$ es la concentración de la especie, v es la tasa de barrido, $\mathrm{R}$ es la constante de gas y $\mathrm{T}$ es la temperatura de la disolución (Rieger, 1994).

La sensibilidad máxima para el electrodo diseñado se obtuvo a una concentración de arsénico de $66 \mu \mathrm{g} / \mathrm{L}$. Sin embargo, se obtuvo un comportamiento lineal para concentraciones superiores a $166 \mu \mathrm{g} / \mathrm{L}$.

Simulación Multifísica del Experimento de Voltametría Cíclica

La simulación computacional de sistemas que funcionan en diferentes dominios físicos permite la predicción de comportamientos, la toma de decisiones y el diseño de experimentos más robustos. Por esta razón, se complementó el experimento de medición y cuantificación de arsénico con una simulación multifísica del electrodo y de la cuantificación por el método de voltametría cíclica, dentro del rango lineal determinado experimentalmente, a fin de comparar los resultados experimentales con los obtenidos en la simulación.

La medición de la voltametría cíclica se simuló utilizando el paquete computacional Comsol Multiphysics ${ }^{\circledR}$ Modeling Software, versión 4.4.
Los coeficientes empleados en la simulación se enumeran a continuación:

Temperatura: $298.15 \mathrm{~K}$

Barrido de Tensión Elétrica: 0.05V/s

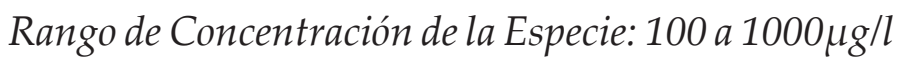

Área Activa del Electrodo: $0.0113 \mathrm{~cm}^{2}$

Coeficiente de Difusión: $6.7 \times 10^{-5} \mathrm{~m}^{2} / \mathrm{s}$

Potencial Inicial (límite inferior): $-0.5 \mathrm{~V}$

Potencial Final (límite superior): $0.8 \mathrm{~V}$

El coeficiente de difusión de la especie bajo análisis se obtuvo a partir de los datos experimentales de voltametría cíclica. Para el módulo de simulación electroquímica disponible en el software utilizado, el $\mathrm{pH}$ es neutral por defecto.

Comsol Multiphysics ${ }^{\circledR}$ versión 4.4 no incluye polímeros conductivos en su repositorio de material, por lo que PEDOT se simuló como una capa conductora estándar. Además, el módulo del simulador asume un potencial de referencia de $0 \mathrm{~V}$, por lo que el electrodo de referencia se omite de la medición.

La Figura 5 muestra la relación lineal teórica entre la densidad de corriente y la concentración de arsénico obtenida de la simulación en Comsol Multiphysics ${ }^{\circledR}$. De la simulación de la prueba de voltametría cíclica se obtuvo curvas de comportamiento para diferentes concentraciones de arsénico con picos de corriente centrados en $0.075 \mathrm{~V}$ aproximadamente. Estas curvas demuestran un comportamiento similar a la forma obtenida en la Figura 2 para los datos experimentales. De los picos de densidad de corriente obtenidos de la simulación para cada concentración de arsénico (Figura 5), es posible calcular el pico de corriente utilizando el área del electrodo $(0.0113 \mathrm{~cm} 2)$, como se muestra en la Tabla 3. 


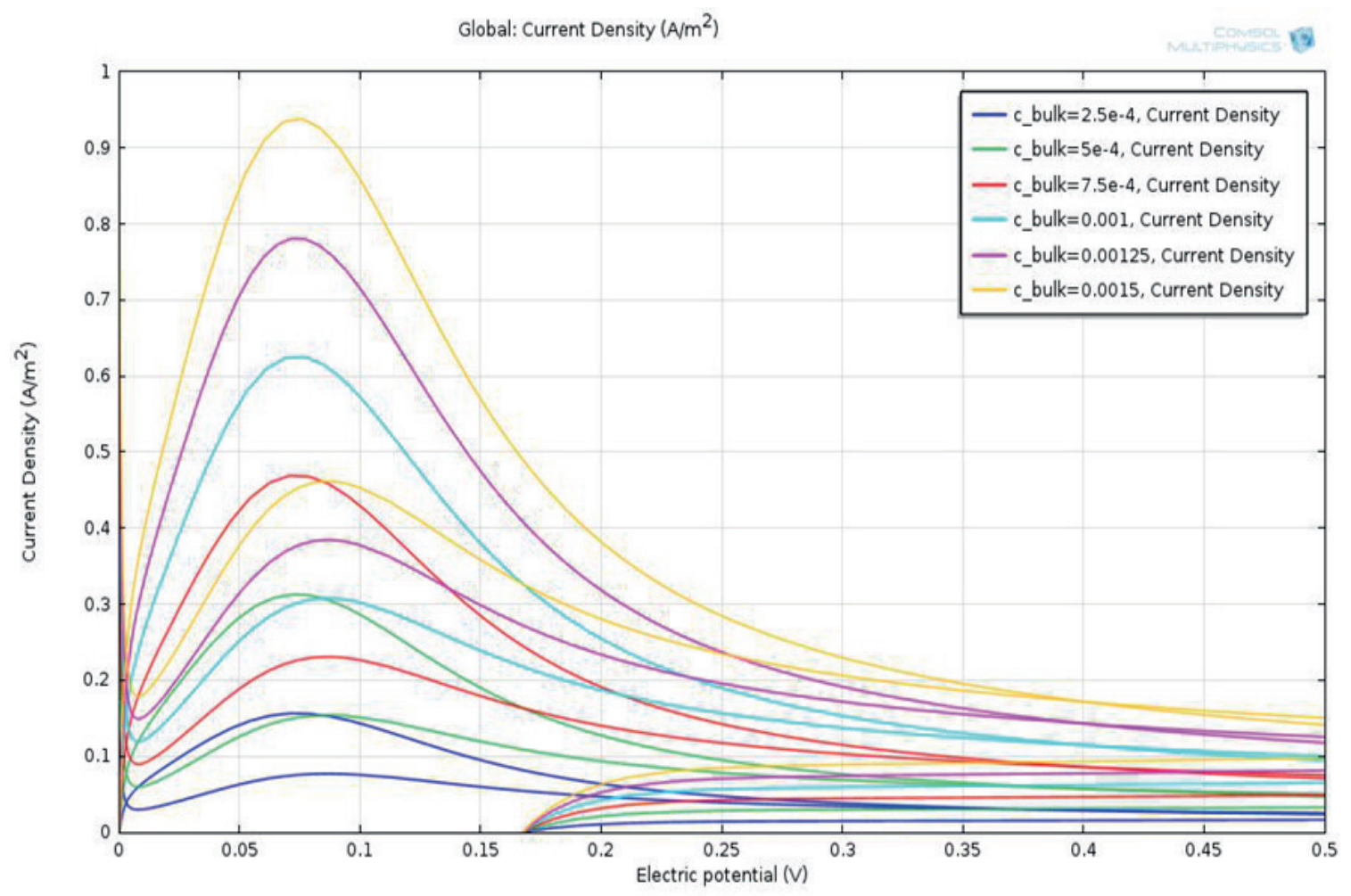

Figura 5. Diagramas Voltametría Cíclica para diferentes concentraciones de arsénico simuladas en Comsol Multiphysics® Modeling Software, versión 4.4. Densidad de corriente $\left(\mathrm{A} / \mathrm{m}^{2}\right)$ vs. Potencial eléctrico (V).

Tabla 3

Picos de corriente. Datos de la simulación

\begin{tabular}{ccc}
\hline Concentración $\mu g / \mathrm{L}$ & $\begin{array}{c}\text { Densidad de Corriente } \\
\mathrm{A} / \mathrm{m}^{2}( \pm 0.03)\end{array}$ & $\begin{array}{c}\text { Pico de Corriente } \\
\mu \mathrm{A}( \pm 0.01)\end{array}$ \\
\hline 167 & 0.23 & 0.26 \\
333 & 0.47 & 0.53 \\
500 & 0.72 & 0.81 \\
667 & 0.93 & 1.05 \\
833 & 1.17 & 1.32 \\
1000 & 1.39 & 1.57 \\
\hline
\end{tabular}


La figura 6 muestra una relación lineal entre el valor del pico de corriente y la concentración de la especie que se deduce a partir de los datos de la Tabla 3.

Los resultados consolidados obtenidos experimentalmente y por medio de la simulación para los picos de corriente obtenidos vs. la concentración de arsénico se muestra en la Figura 7. La relación lineal para ambos casos es consistente, aunque existe una diferencia en el valor de la corriente de $50 \mu \mathrm{A}$ entre los datos experimentales y los simulados. Esta diferencia es sistemática, puesto que se presenta para todas las mediciones. Para determinar su origen se necesita de mayor información acerca de las características de la capa conductora estándar que está disponible en el repositorio del simulador multifísico.

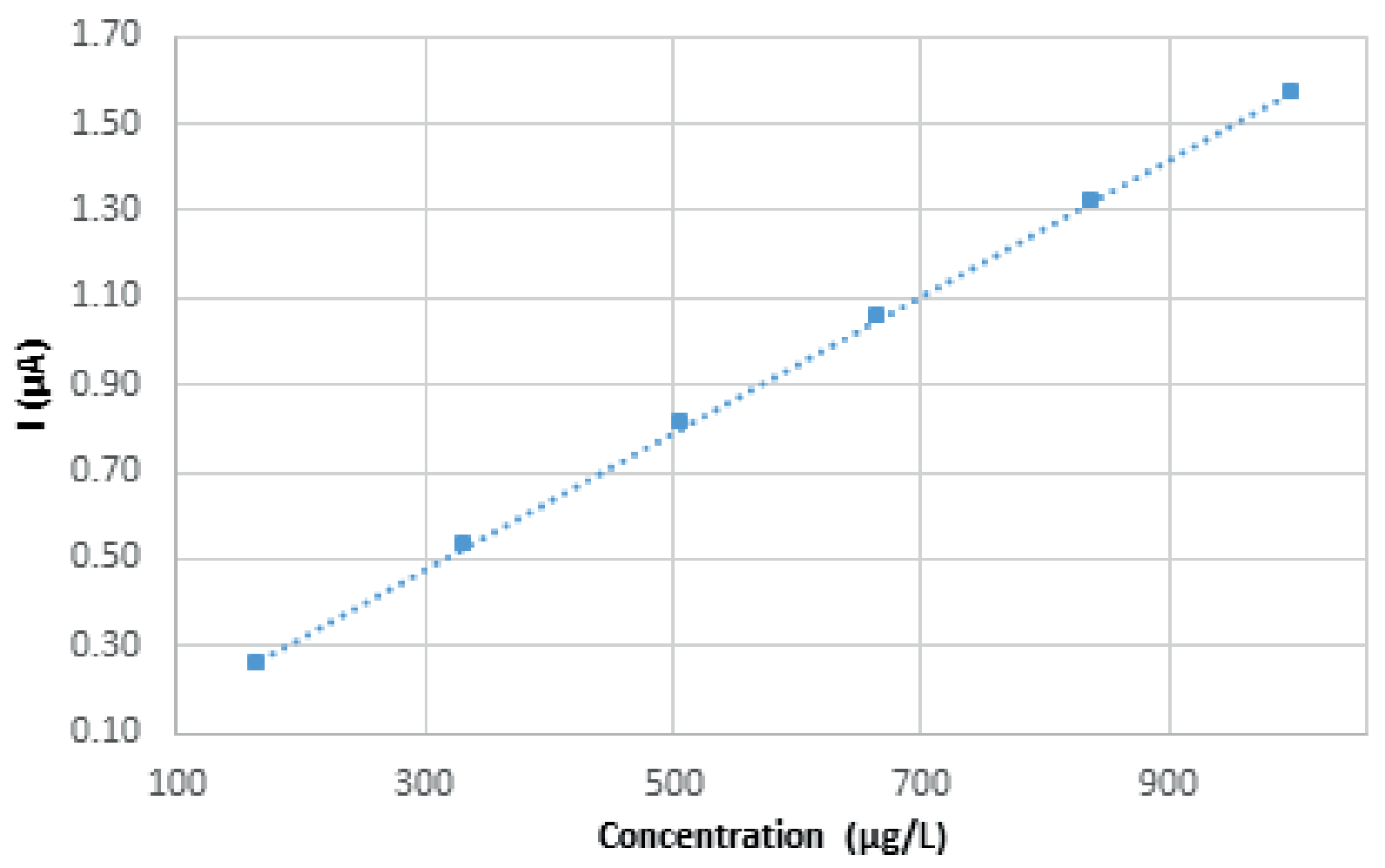

Figura 6. Picos de corriente obtenidos de la simulación de voltametría cíclica en Comsol Multiphysics ${ }^{\circledR}$ Modeling Software, versión 4.4. Pico de corriente $(\mu \mathrm{A})$ vs Concentración de arsénico $(\mu \mathrm{g} / \mathrm{L})$. 


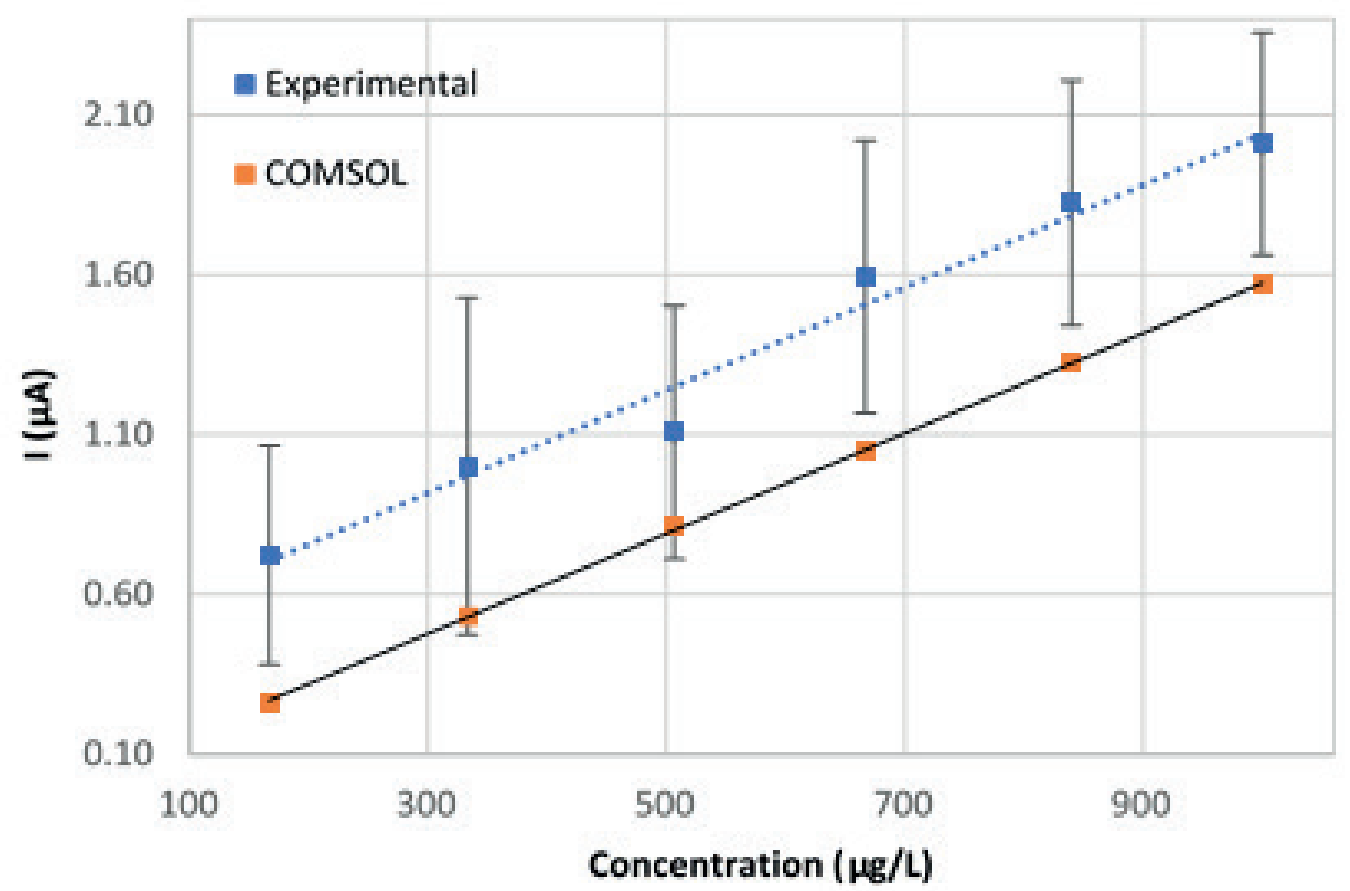

Figura 7. Pico de corriente $(\mu \mathrm{A})$ vs Concentración de arsénico $(\mu \mathrm{g} / \mathrm{L})$. Datos consolidados: experimentales y simulados.

\section{Conclusiones}

Se demostró que el arsénico en disolución acuosa puede ser cuantificado por el electrodo de oro recubierto con PEDOT utilizando voltametría cíclica, con una sensibilidad a variaciones de corriente a partir de $66 \mu \mathrm{g} / \mathrm{L}$ y un rango lineal de 166 a $1000 \mu \mathrm{g} / \mathrm{L}$. Mediante un análisis ANOVA se confirmó que la amplitud del pico de corriente de oxidación para el arsénico es proporcional a la concentración de la especie en la disolución. Se calculó un coeficiente de difusión de $6.7 \times 10^{-5} \mathrm{~m}^{2} / \mathrm{s}$ para el arsénico a partir de los datos generados experimentalmente por voltametría cíclica en condiciones de laboratorio controladas. Además, el experimento se reprodujo con éxito en Comsol Multiphysics ${ }^{\circledR}$ Modeling Software, versión 4.4, utilizando el coeficiente de difusión calculado a partir de los datos experimentales.
Los resultados de la simulación podrían mejorarse al incluir el polímero conductor como parte de la configuración, pero este tipo de material no está disponible como estándar en el repositorio de materiales COMSOL. Se recomienda crear el material de manera personalizada a partir de los datos que facilita el fabricante.

La cuantificación de muestras de campo con el electrodo desarrollado permitiría extender el alcance de este experimento. Para ello se sugiere utilizar un potenciostato portátil o diseñar un generador de barrido ajustable.

Los resultados actuales pueden mejorarse mediante la adición de nanopartículas dentro de la capa de polimerización conductiva del electrodo, tales como nanopartículas de hierro o nanotubos. 


\section{Reconocimientos}

Al Tecnológico de Costa Rica (ITCR) y al Consejo Nacional de Rectores (CONARE) por el apoyo financiero brindado a través del Fondo Especial para la Educación Superior (FEES). A los laboratorios CEQIATEC y CIPA, a la Escuela de Ingeniería en Ciencia de Materiales, a Carlos FabiánCotodelaEscueladeIngeniería Electrónica del ITCR, a la CICIMA de la Universidad de Costa Rica y a todos los estudiantes asistentes y profesionales relacionados con este proyecto, especialmente a María Angélica Badilla, Priscila Hernández, Betzabeth Zúñiga y Federico Masís.

\section{Referencias}

Andrienko, D. (2008). Cyclic Voltammetry. Chapter 1, 1-12. Recuperado de https://doi. org/10.1016/j.jelechem.2004.03.028

Chen, T. W., Tsai, T. H., Chen, S. M., y Lin, K. C. (2011). Using PEDOT film modified electrode to monitor iodide and its enhancement of arsenite sensing. International Journal of Electrochemical Science, 6(6), 2043-2057.

Dai, X., y Compton, R. G. (2006). Detection of As(iii) via oxidation to As(v) using platinum nanoparticle modified glassy carbon electrodes: arsenic detection without interference from copper. Analyst, 131(4), 516521. Recuperado de https://doi.org/10.1039/ B513686E

Defensoría de los Habitantes de la República (2014). Informe de avance de la investigación de oficio sobre el problema de contaminación del agua con arsénico en poblados de Guanacaste y la zona norte del país. Mayo 2014, 1-21. Recuperado de http://www.dhr. go.cr/informacion relevante/resoluciones/ informe arsenico.pdf

Gomez-Caminero, A., Howe, P., Hughes, M., Kenyon, E., Lewis, D.R., Moore, M., Ng, J.
C., Aitio, A., y Becking, G. (2000). A Environmental Health Criteria 224 Arsenic and Arsenic Compounds. Geneva: World Health Organization

Hayt, W. H., Kemmerly, J. E., y Durbin, S. M. (1986). Engineering circuit analysis. New York: McGraw-Hill

Kumar Jena, B., y Retna Raj, C. (2008). Gold Nanoelectrode Ensembles for the Simultaneous Electrochemical Detection of Ultratrace Arsenic, Mercury, and Copper. Analytical Chemistry, 80(13), 4836-4844. Recuperado de https://doi.org/10.1021/ac071064w

Litter, M.I., Armienta, M.A., y Farias, S. (2009). Metodologías analíticas para la determinación y especiación de arsénico en aguas y suelos. Buenos Aires: IBEROARSEN, CYTED. Recuperado de https://limza.cl/jdownloads/Libros/ metodologas analticas para la determinacin $\mathrm{y}$ especiacin de arsnico en aguas $\mathrm{y}$ suelos.pdf

Mora-Alvarado, D., Urbina-Campos, A., y Chamizo-García, H. (2015). Estudio ecológico sobre insuficiencia renal crónica y arsénico en las aguas para consumo humano por distritos en Guanacaste. Revista Tecnología en Marcha, 28(2), pág. 102-115. Recuperado de http://revistas.tec.ac.cr/index.php/ tec marcha/article/view/2337/2126

Morand, E., Giménez, M., Benitez, M., y Garro, O. A. (2008). Determinación de arsénico en agua por espectrometría de absorción atómica con generación de hidruro (HGAAS). Facultad de Agroindustrias, Universidad Nacional del Nordeste (UNNE), 4. Recuperado de http://www.unne.edu.ar/unnevieja/ Web/cyt/cyt/2002/08-Exactas/E-048.pdf

Quansah, R., Armah, F. A., Essumang, D. K., Luginaah, I., Clarke, E., Marfoh, K., ... Dzodzomenyo, M. (2015). Association of arsenic with adverse pregnancy outcomes/infant 
mortality: A systematic review and meta-analysis. Environmental Health Perspectives, 123(5), 412-421. Recuperado de https:// doi.org/10.1289/ehp.1307894

Quijandria, G., Berrocal, J.; Pratt, L. (1997). La industria de la piña en Costa Rica. Análisis de Sostenibilidad. Recuperado de https://doi. org/10.13140/RG.2.1.1024.0082

Rieger, P. (1994). Electrochemistry. New York: Chapman \& Hall.

Rodríguez, E., y Desan, M. (2016). Prueba de Chi-Cuadrada. Universidad Nacional Autónoma de México, Facultad de Estudios Superiores Cuautitlán. Recuperado de http://asesorias.cuautitlan2.unam.mx/ Laboratoriovirtualdeestadistica/CARPETA3INFERENCIA ESTADISTICA/DOC INFERENCIA/TEMA4/08PRUEBADECHICUADRADA.pdf

Salimi, A., Mamkhezri, H., Hallaj, R., y Soltanian, S. (2008). Electrochemical detection of trace amount of arsenic(III) at glassy carbon electrode modified with cobalt oxide nanoparticles. Sensors and Actuators B Chemical, 129 (1), 246-254. Recuperado de https://doi.org/http://dx.doi.org/10.1016/j. snb.2007.08.017
Starbird, R., Bauhofer, W., Meza-Cuevas, M., y Krautschneider, W. H. (december, 2012). Effect of experimental factors on the properties of PEDOT-NaPSS galvanostatically deposited from an aqueous micellar media for invasive electrodes. Conferencie paper in $V$ Biomedical Engineering International Conference (BMEiCON), Ubon Ratchathani, Thailand.

Tanaka, M., Takahashi, Y., Yamaguchi, N., Kim, K.-W., Zheng, G., y Sakamitsu, M. (2013). The difference of diffusion coefficients in water for arsenic compounds at various $\mathrm{pH}$ and its dominant factors implied by molecular simulations. Geochimica et Cosmochimica Acta, 105, 360-371.

World Health Organization. (2004). Arsenic in drinking-water. IARC Monographs on the Evaluation of Carcinogenic Risks to Humans, 84, 41-267. Recuperado de https://doi.org/10.1016/j.kjms.2011.05.002

World Health Organization (2010) Exposure to Arsenic: a major public health concern. Geneva. Recuperado de https://www.who.int/ ipcs/features/arsenic.pdf?ua=1

Recibido: 23 de agosto de 2018 Aceptado: 23 de noviembre de 2018 